\title{
SOFTWARE EDUCATIVO Y SU IMPORTANCIA EN EL PROCESO ENSEÑANZA-APRENDIZAJE
}

\author{
AUTORES: Kirenia Maldonado Zuñiga. ${ }^{1}$ \\ Raquel Vera Velázquez. ${ }^{2}$ \\ Lisbeth Mercedes Ponce Delgado 3 \\ Franklin Jhimmy Tóala Arias ${ }^{4}$
}

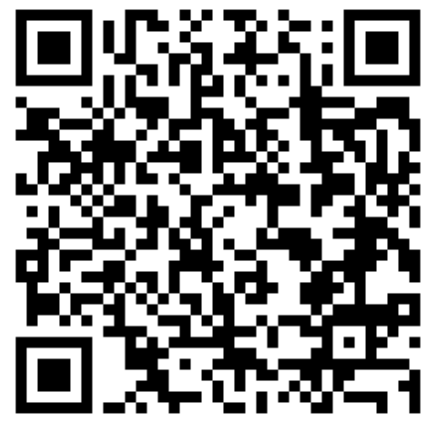

\section{DIRECCIÓN PARA CORRESPONDENCIA:kirenia.maldonado@unesum.edu.ec}

Fecha de recepción: 25/01/2020

Fecha de aceptación: 22/02/2020

RESUMEN

Para garantizar un mayor aprendizaje en los estudiantes, es importante utilizar software educativo en las clases. El objetivo de esta investigación fue dar a conocer los beneficios que aporta el uso de estas herramientas en el proceso de enseñanza aprendizaje. Las tecnologías exigen romper esquemas tradicionales y brindar conocimientos que implican un acercamiento del sujeto y el objeto que va más allá de lo presencial. La importancia radico en el aprendizaje autónomo y el desarrollo de ciertas habilidades cognitivas. Estos programas didácticos interactivos, permitieron el cumplimiento de los objetivos de las asignaturas, el docente valorara qué necesidad existe en su asignatura y promueve un mayor aprendizaje. Se utilizaron métodos científicos como, histórico lógico, análisis - síntesis, observación y revisión bibliográfica. Los resultados comprobaron que la utilidad del software educativo en las clases debe ser una prioridad. Los estudiantes aprenden a utilizar todas las herramientas necesarias y se crea un entorno agradable. El software aplicado en la educación constituye un invalorable recurso didáctico que debe ser explotado por docentes y estudiantes, estos se retroalimentan y facilita una atención diferenciada. Las clases son amenas y motivadoras por lo que se logra una concentración en los estudiantes y esta desarrolla el pensamiento. Se concluye con los cambios significativos que causaron la utilización del software educativo en las clases, orientado por el docente. Es apreciable porque facilitó el trabajo según las diferencias individuales y el desarrollo de los aspectos cognitivos de los estudiantes.

PALABRAS CLAVE: conocimiento; docencia; estudiantes; habilidades; tecnología.

\section{EDUCATIONAL SOFTWARE AND ITS IMPORTANCE IN THE TEACHING- LEARNING PROCESS}

\footnotetext{
${ }^{1}$ Universidad Estatal del Sur de Manabí, Facultad de Ciencias Técnicas, Jipijapa, Manabí, Ecuador

2 Universidad Estatal del Sur de Manabí, Facultad de Ciencias Naturales y de la Agricultura

${ }^{3}$ Licenciada de Ciencia de la Educación. Universidad Laica Eloy Alfaro de Manabí.

${ }^{4}$ Universidad Estatal del Sur de Manabí, Facultad de Ciencias Técnicas, Jipijapa, Manabí,
} 
Kirenia Maldonado Zuñiga., Raquel Vera Velázquez., Lisbeth Mercedes Ponce Delgado...

\section{ABSTRACT}

To ensure greater student learning, it is important to use educational software in class. The objective of this research was to publicize the benefits of using these tools in the teaching-learning process. Technologies require breaking traditional patterns and providing knowledge that implies an approach of the subject and the object that goes beyond the face-to-face. The importance lies in autonomous learning and the development of certain cognitive skills. These interactive didactic programs, allowed the fulfillment of the objectives of the subjects, the teacher valued what need exists in his subject and promotes greater learning. Scientific methods such as, historical - logical, analysis - synthesis, observation and bibliographic review were used. The results verified that the usefulness of educational software in class should be a priority. Students learn to use all the necessary tools and a pleasant environment is created. The software applied in education constitutes an invaluable didactic resource that must be exploited by teachers and students, they feed back and provide differentiated attention. The classes are enjoyable and motivating so that a concentration is achieved in the students and this develops thinking. It concludes with the significant changes that caused the use of educational software in the classes, oriented by the teacher. It is appreciable because I facilitate the work according to the individual differences and the development of the cognitive aspects of the students.

KEYWORDS: knowledge; skills; students; teaching; technology.

\section{INTRODUCCIÓN}

En la actualidad, varios países tienen avances significativos con el uso de las tecnologías, conquistando fácilmente todos los sectores en especial la educación. El software educativo puede utilizarse en todos los niveles instructivos. Las transformaciones digitales hacen parte de la vida cotidiana del ser humano, a través de procesos, métodos y estrategias que favorecen al conocimiento y habilidades de los estudiantes. Todo esto es posible con la innovación y la implementación de las tecnologías.

La utilización del software educativo en el proceso enseñanza- aprendizaje en las instituciones educativas, establece una base significativa en las clases de las diferentes asignaturas lo que permite una interacción dinámica entre el docente y el estudiante, se puede observar el cambio favorable que estas causan en el aprendizaje de los estudiantes, las clases son motivadoras, lo que estimulan a la efectividad y eficacia del conocimiento en el proceso de enseñanza- aprendizaje. (Bezanilla, M. J 2010).

El Informe Mundial sobre la Educación de la (UNESCO, 2004) indica que "Los docentes y la enseñanza en un mundo en mutación", se describió que el impacto de las tecnologías en los métodos tradicionales de enseñanza - aprendizaje, prediciendo la transformación de este proceso y la forma en la que los docentes deben prepararse con estas herramientas.

La importancia del software educativo es que está destinado a la enseñanza y el aprendizaje autónomo, además, permite el desarrollo de ciertas habilidades cognitivas. Cuando se habla del software educativo, se hace referencia a los programas educativos o programas didácticos interactivos, conocidos también, creados con la finalidad específica de ser utilizados para facilitar los procesos de enseñanza y aprendizaje. Se excluyen de este tipo de programas, todos aquellos de 
uso general utilizados también en las unidades educativas como: procesadores de texto, gestores de base de datos, hojas de cálculo, editores gráficos, entre otros. (Bezanilla, M. J, 2010)

El software educativo se define por ser bien interactivo, las herramientas multimedia, videos, sonidos, fotografías, diccionarios especializados, ejercicios y juegos instructivos que apoyan las funciones de evaluación y diagnóstico todo esto con las explicaciones de docentes logran una buena combinación para elevar el conocimiento. El objetivo es que el intercambio aporte un aprendizaje significativo. (Bezanilla, M. J, 2010)

Ecuador cuenta con las tecnologías y sus herramientas en una gran mayoría de los centros educativos. La época en que se vive se puede evidenciar las evoluciones que están surgiendo en la educación, es una necesidad de educar y aprovechar los beneficios que estos medios brindan. La utilización de software educativo, según las necesidades de los estudiantes, les genera un conocimiento preciso. Es evidente que cuando el docente utiliza software los estudiantes se concentran más y adquieren el conocimiento de una forma placentera lo que permite un aprendizaje interactivo y dinámico. (García, F, 1997)

\section{DESARROLLO}

Es importante considerar el uso del software educativo con metodologías adecuadas en las diferentes clases, estos estimulan el interés a través de nuevas experiencias que dan origen al conocimiento y al pensamiento, de esta forma se favorecen los estudiantes con habilidades, conocimiento y dominio en las tecnologías para aumentar su potencial. Plantear estrategias de enseñanza que fortalezcan la educación en los diferentes niveles y que a su vez promuevan aprendizajes significativos, en el cual los estudiantes puedan tener una participación activa en las clases, así como replicar esos conocimientos en la práctica.

Los sistemas educativos en la actualidad enfrentan la utilización de las tecnologías de la información y la comunicación en el proceso enseñanza como medio de enseñanza en sus clases, estas propician una mayor motivación, dedicación e interacción. En los tics se destaca el software educativo como medio educativo, estas cumplen un papel importante en la educación, garantiza la preparación de los docentes, en la didáctica, la metodología y la utilización de estas herramientas para el apoyo del proceso de enseñanza - aprendizaje. (Gates, B, 1997)

Existen numerosas investigaciones sobre la evolución del conocimiento y el aprendizaje en los educandos, las diferentes situaciones que se presentan en las clases demuestran la necesidad de utilizar herramientas que faciliten el conocimiento a los estudiantes, así como promover una enseñanza - aprendizaje interactivo y práctico. Es necesario realizar un cambio efectivo que permita un mayor aprendizaje, lo cual permitirá tomar acciones tendientes a mejorar y corregir los posibles errores, con el objetivo de lograr una visión general de la situación actual de la enseñanza y aprendizaje en las diferentes instituciones educativas.

La clase con software educativo es aquella cuyo objetivo se corresponde con la asignatura que se imparte y el uso de este medio de enseñanza es fundamental, que contribuye a la asimilación de los contenidos y se concreta en tareas docentes dirigidas a la búsqueda, selección procesamiento interactivo y conversación de la información usando medios informáticos. (Bezanilla, M. J, 2010) 
Kirenia Maldonado Zuñiga., Raquel Vera Velázquez., Lisbeth Mercedes Ponce Delgado...

La finalidad de la clase con software educativo es lograr que los estudiantes venzan los objetivos, esto conlleva a seleccionar para la clase el software educativo necesario. En función del objetivo se diseña las tareas docentes (para la clase o como trabajo autónomo). El enunciado de las tareas docentes debe contener las orientaciones mínimas necesarias acerca de la navegación por el software educativo de modo que el estudiante no se pierda en el entorno. Las tareas docentes deben corresponder a diferentes niveles de asimilación, ser desarrolladoras y diferenciadas para su evaluación. (Trevejo Alonso, J. A, 2012)

El aprendizaje con ayuda del software educativo desarrolla habilidades, pero para lograr un aprendizaje significativo es necesario construir una interacción fuerte entre estos componentes, la parte teórica y las prácticas estas deben ser guiadas por el docente para ganar en precisión y potencia, de esta forma surgen los cambios favorables.

El proceso de enseñanza - aprendizaje, es una de las principales preocupaciones generalizadas en los diferentes niveles educativos, se han realizado una gran variedad de estrategias $\mathrm{y}$ planteamientos didácticos para mejorar este proceso, sin embargo, en algunos centros educativos el enfoque dominante es el aprendizaje tradicional.

Como medio de enseñanza ha generado entre otras necesidades la preparación de los docentes en ejercicio, la elaboración de guías o metodologías de selección de software educativo para constatar la pertinencia de su incorporación en las unidades educativas y la capacitación para medir su valor de uso en la toma de decisiones en cuanto a su empleo sistemático.

Recopilan un amplio volumen de información acerca de los conocimientos que se trabajan en las diferentes asignaturas, pero en algunos contenidos la ofrecen de forma acabada o enciclopédica con un modelo o imagen que refleja la esencia del contenido, como si se pasara un texto por la pantalla que concreta las características, las relaciones o nexos esenciales del contenido de forma general.

La enseñanza en todos los aspectos debe desarrollarse dinámica e interconectada de forma interactiva. La investigación tiene su importancia educativa puesto que proyecta, impulsar y estimular a los docentes a realizar investigaciones donde garantice la realidad objetiva que contribuye a crear nuevas ideas, aportes, para la solución de los problemas dentro de un contexto real de una comunidad educativa según las insuficiencias y características de los estudiantes, a la vez se crea la investigación participativa y con experiencias educativas. Es un eje fundamental para transformar el desarrollo de los procesos de aprendizaje, convirtiéndose en un proceso de actividad dinámica, que pretende una acción en los patrones de programas, que enmarque dentro del contexto realista acorde con las necesidades e intereses de la presente exigencia en la educación. (Hernández, S., Roberto, Fernández, Carlos, C., \& Baptista, L. P, 2000)

Algunas características del software educativo que benefician el desarrollo de la atención a la diversidad: carácter no lineal posibilita que cada estudiante pueda elegir su camino de aprendizaje, según sus intereses y posibilidades. Ritmo de navegación: cada estudiante puede navegar a su ritmo, unos necesitarán más tiempo que otros en procesar la información presentada. Estilos de aprendizaje: el estudiante puede optar por estilos de aprendizaje diferentes. Ascender de la teoría a la práctica, comenzar a partir de problemas surgidos y buscar soluciones heurísticas. Adaptabilidad: a las características individuales del aprendiz. (Bezanilla, M. J, 2010) 
El software educativo también propicia un material elaborado para uso didáctico. Se utiliza el ordenador, como soporte en el que los estudiantes realizan las actividades. Estas actividades son interactivas, contestan inmediatamente las acciones de los estudiantes y permiten un diálogo y un intercambio de informaciones entre el ordenador y éstos. Individualizan el trabajo, se adaptan al ritmo de trabajo de cada estudiante y las actividades se pueden adaptar según las necesidades de cada estudiante. Son fáciles de utilizar y los conocimientos informáticos necesarios para utilizar el software son básicos. (Bezanilla, M. J, 2010)

Las funciones de estos softwares dependen del uso que se le dé y de la forma que se utilice, su funcionalidad, depende de las ventajas y los inconvenientes que esté presente para su uso, el resultado se obtendrá según las características del material escogido y de su adecuación al contexto educativo al que se aplica, también es importante el modo en que el docente establezca su utilización.

Según (Guzmán, J, 2011) estas herramientas favorecen el proceso enseñanza aprendizaje y son utilizadas por docentes y estudiantes, que logran una clase participativa interactiva, ya sea individual o colectiva, lo que proporciona un conocimiento satisfactorio. Este medio resulta un instrumento de efectiva utilidad para el desarrollo de la interacción y habilidades específicas de cada nivel de enseñanza. Contribuyen a elevar la calidad del aprendizaje y posibilita una mejor atención al tratamiento de las diferencias individuales en correspondencia del diagnóstico de los educandos.

Beneficios que brinda el software educativo en el aula: permite la interactividad con los estudiantes, estos se retroalimentan y evalúan lo aprendido automáticamente, las representaciones animadas atraen su motivación, desarrollan habilidades en la signatura que trabajen el software e informáticas, realizan procesos complejos, facilita un trabajo diferenciado, los estudiantes trabajan independiente, desarrollan el pensamiento, la imaginación, la creatividad y la memoria, transmite gran cantidad de información en poco tiempo. (Sánchez, J, 2009)

El docente cuenta con varias ventajas al utilizar los software educativos, estos se engrandecen en el campo de la Pedagogía al unir la tecnología y los métodos de enseñanza aprendizaje, organiza una clase atractiva y dinámica, los software se pueden adaptar a las características y necesidades de los estudiantes teniendo en cuenta el diagnóstico en el proceso de enseñanza aprendizaje, lo cual permite elevar su conocimiento, controla de las tareas de forma individual o colectiva, se evidencia la interdisciplinariedad de las diferentes asignaturas, de esta forma el docente no se queda en las clases tradicionales. (Telepieza, 2008)

Los softwares educativos están creados con metodologías que permita a los estudiantes desarrollar habilidades y conocimientos necesarios para combatir las dificultades presentadas en clases, estos softwares la gran mayoría están diseñados en una plataforma con tecnología compatible para los sistemas operativos, amigable y fácil de manejar para los estudiantes, la cual permita el desarrollo de las actividades interactivas.

Según (Sánchez, J., Iriarte, P., \& Mendez, M, 2000) estos pueden ser usados durante la clase, dentro del contexto estudiantil, o incluso en un local externo a este ambiente, como en casa, estos son una herramienta innovadora que despiertan el interés del estudiante facilitando la adquisición del 
Kirenia Maldonado Zuñiga., Raquel Vera Velázquez., Lisbeth Mercedes Ponce Delgado...

conocimiento y permitiendo una fijación del contenido más permanente. Su variedad permite captar la atención de los estudiantes, manteniéndolos motivados e interesados por un largo periodo.

El objetivo de esta investigación es dar a conocer la importancia y los beneficios que aporta la utilización del software educativo en el proceso de enseñanza aprendizaje. El uso de las TIC en las diferentes asignaturas exige romper esquemas tradicionales y brindan conocimientos que implican un acercamiento del sujeto y el objeto que va mucho más allá de lo presencial. Los procesos de utilizar las tecnologías inciden de manera favorable en el proceso de enseñanzaaprendizaje.

La importancia de la utilización del software educativo es por lo que favorece el aprendizaje, efectivo que aporta en el ámbito del conocimiento. Es necesario que los docentes asuman como una meta, el buscar estrategias para que todo estudiante desarrollen habilidades con el uso del software educativo. Los docentes son los responsables de usar metodologías que contribuyan a la construcción del aprendizaje en los estudiantes de una forma responsable e interesante.

\section{MATERIALES Y MÉTODOS}

La utilización de varios métodos científicos y técnicas para la búsqueda de información, evidenciaron un diagnóstico para valorar la importancia de utilizar el software educativo en las clases.

Del nivel teórico:

Histórico - lógico: se usó en la construcción de la investigación con énfasis en la búsqueda de los antecedentes, importancia y función del software educativo.

Análisis - síntesis: se utilizó para determinar el uso del software educativo en las clases.

Del nivel Empírico:

Observación: permitió investigar y analizar la mayor cantidad de información, dando las pautas necesarias para el desarrollo de la investigación.

Revisión bibliográfica: Se utilizó para la recopilación de información relacionada al tema, mediante libros, revistas de carácter científico, internet, entre otros

\section{RESULTADOS}

Los resultados alcanzados en la investigación demostraron que la utilización del software educativo en las clases debe ser una prioridad. Los estudiantes aprenden a utilizar todas las herramientas posibles. El software aprovechado en la educación constituye un invalorable recurso didáctico que debe ser explotado por estudiantes y docentes. Las exigencias de la actividad docente en la que se emplee el software educativo para la formación del conocimiento, requiere de la preparación sistemática de los docentes en aras de lograr su desarrollo integral y la búsqueda de una buena clase, en este sentido, el software educativo propicia la sistematización de los contenidos impartidos en las diferentes asignaturas si se utiliza como medio de enseñanza. Una de las principales ventajas del software educativo es que posibilitan la interactividad con los estudiantes, 
retroalimentándolos y evaluando lo aprendido, facilita una atención diferenciada con los estudiantes, estos desarrollan habilidades en el trabajo con los medios computarizados. Las clases son amenas y motivadoras por lo que se logra una concentración en los estudiantes y esta les facilita el desarrollo del pensamiento y el conocimiento, logrando una cultura general integral en los educandos.

\section{CONCLUSIONES}

Se concluyó con los cambios significativos que se produjeron al utilizar el software educativo en las aulas orientado por el docente. Es valioso en la educación porque facilitó el desarrollo del trabajo independiente del estudiante. Estos son un recurso didáctico que se deben utilizar dentro de un proyecto pedagógico definido. El software educativo constituye una excelente fuente para aprender y adquirir conocimientos, estos generalmente establecen los nexos fundamentales que conforman la información de la que son portadores, provocando que los docentes interpreten como acabado el proceder metodológico que conduce a la formación del conocimiento, desarrollo de habilidades, realización de actividades y pasen después de la presentación del modelo expresado por estos medios a la fijación del contenido. Esta herramienta interactiva ayuda a los estudiantes, ellos sienten que están jugando, pero en realidad se están nutriendo de conocimientos. En varias instituciones educativas se está implementado el uso de software, porque son considerados como un poderoso medio para el desarrollo de las diferencias individuales y desarrollo de los aspectos cognitivos del alumno. El uso de software debe verse como un elemento dinamizador del aprendizaje que conduzcan a los estudiantes por el proceso del conocimiento. En los últimos tiempos varios docentes están utilizando el software educativo, debido que el estudiante mantiene un aprendizaje interactivo.

\section{REFERÉNCIAS BIBLIOGRÁFICAS}

Bezanilla, M. J. (2010). Bases técnico-pedagógicas para la elaboración de software educativo. Universidad Nacional de Educación a Distancia, pp. 164-167.

García, F. (1997). Organización escolar y gestión de centros educativos. Málaga: Algibe.

Gates, B. (1997). Camino al futuro. España: MCGRAW-HILL S.A. Guzmán, J. (03 de febrero de 2011). Las TIC y la crisis de la educación. Obtenido de http://www.virtualeduca.org/documentos/yanez.pdf

Guzmán, J. (03 de febrero de 2011). Las TIC y la crisis de la educación. Obtenido de http://www.virtualeduca.org/documentos/yanez.pdf

Hernández, S., Roberto, Fernández, Carlos, C., \& Baptista, L. P. (2000). Metodología de la investigación. Colombia: Mc Graw Hill.

Sánchez, J. (2009). Plataformas de enseñanzas virtuales para entornos educativos. Obtenido de Revista de Medios y Educación: http://www.sav.us.es/pixelbit/pixelbit/articulos/n34/15.pdf

Sánchez, J., Iriarte, P., \& Mendez, M. (2000). Construyendo y Aprendiendo con elcomputador. Integración de medios interactivos para la capacitación de profesores en informática educativa. VIII Congreso Nacional de Informática Educativa. Chillan: Universidad del Bio Bio.

Telepieza. (20 de diciembre de 2008). Los diferentes tipos de portales. Obtenido de http://www.telepieza.com/wordpress/2008/01/29/los-diferentes-tipos-de-portales-queexisten-en-internet/

Trevejo Alonso, J. A. (2012). Joomla! La web en entornos educativos. Obtenido de Instituto Nacional de Tecnologías Educativas $\quad y$ Formación del Profesorado: http://www.ite.educacion.es/formacion/materiales/99/cd/mod_01/caractersticas_de_jo omla.html 
Kirenia Maldonado Zuñiga., Raquel Vera Velázquez., Lisbeth Mercedes Ponce Delgado... 